Article

\title{
Magnetic Properties and the Superatom Character of 13-Atom Platinum Nanoclusters
}

\section{Emil Roduner ${ }^{1,2, *}$ and Christopher Jensen ${ }^{1, \dagger}$}

1 Institute of Physical Chemistry, University of Stuttgart, Pfaffenwaldring 55, D-70569 Stuttgart, Germany; E-Mail: christopher.jensen@thyssenkrupp.com

2 Chemistry Department, University of Pretoria, Pretoria 0002, South Africa

$\dagger$ Present address: ThyssenKrupp Industrial Solutions AG, Business Unit Process Technologies, Neubeckumer Straße 127, D-59320 Ennigerloh, Germany.

* Author to whom correspondence should be addressed; E-Mail: e.roduner@ipc.uni-stuttgart.de or emil.roduner@up.ac.za; Tel.: +0041-44-422-34-28.

Academic Editor: Carlos J. Gómez García

Received: 25 October 2015 / Accepted: 18 November 2015 / Published: 26 November 2015

\begin{abstract}
: 13-atom platinum nanoclusters have been synthesized quantitatively in the pores of the zeolites $\mathrm{NaY}$ and KL. They reveal highly interesting magnetic properties like high-spin states, a blocking temperature, and super-diamagnetism, depending heavily on the loading of chemisorbed hydrogen. Additionally, EPR active states are observed. All of these magnetic properties are understood best if one considers the near-spherical clusters as analogs of transition metal atoms with low-spin and high-spin states, and with delocalized molecular orbitals which have a structure similar to that of atomic orbitals. These clusters are, therefore, called superatoms, and it is their analogy with normal atoms which is in the focus of the present work, but further phenomena, like the observation of a magnetic blocking temperature and the possibility of superconductivity, are discussed.
\end{abstract}

Keywords: superatom concept; $\mathrm{Pt}_{13}$ nanoclusters; shell structure; hyperfine couplings; high-spin states; super-diamagnetism; granular superconductivity 


\section{Introduction}

Platinum is not among the elements which exhibit long-range ferromagnetic order in the bulk. However, it is well known that ferromagnetism is greatly affected when the dimensionality of the material is reduced to thin films or monoatomic chains [1], and in nanoparticles, elements which are typically diamagnetic in the bulk, like $\mathrm{Au}, \mathrm{Pd}$, and $\mathrm{Pt}$, develop an increasingly higher magnetic moment with decreasing size $[2,3]$ and show high-spin states in well-defined clusters [4,5]. Less well known, although predicted [6] but observed experimentally for the first time only recently [7], is the fact that the diamagnetic moment per atom increases dramatically in small clusters. An understanding of these size-dependencies is of considerable interest for fundamental reasons.

Here we focus on the magnetic properties of Pt nanoclusters which can be prepared quantitatively in the pores of zeolites in a size of $13 \pm 2$ atoms, as derived from Electron Paramagnetic Resonance (EPR) [8,9] and Extended X-ray Absorption Fine Structure (EXAFS) experiments [10,11]. Rather than limited by the free diameter of the pores, the cluster size is given by the special stability of the geometrically-closed shell with one atom in the center that is surrounded by 12 atoms in the first shell, which corresponds to the maximum coordination number of close packed spheres. A small fraction of the clusters $(\approx 0.03 \%$ of the total $\mathrm{Pt}$ in the sample) is EPR-active after hydrogen adsorption, showing hyperfine splitting due to 12 equivalent Pt nuclei. The EPR signal disappears on hydrogen desorption. $15 \%-20 \%$ of the Pt occurs in a high-spin state with a magnetic moment of $3.7 \pm 0.4 \mu$ B per cluster, depending somewhat on hydrogen coverage, the rest is diamagnetic [10,11]. Based on EPR and EXAFS experiments the structure is compatible with close to icosahedral symmetry, perhaps slightly distorted, owing to the open-shell nature of the cluster, but more recent quantum chemical calculations predict the buckled biplanar isomer to be more stable by as much as $1.5 \mathrm{eV}$ [12], and this structure may still be compatible with the EXAFS data but not with EPR. Hydrogen can be adsorbed and desorbed reversibly, but on reaction with $\mathrm{CO}$ the cluster disintegrates irreversibly to $\mathrm{Pt}_{2}(\mathrm{CO})_{5}$ complexes [13].

Particularly fruitful for an understanding of the observed phenomena is the description of these near-spherical clusters as superatoms, a concept analogous to atoms with discrete energy states, with atomic orbitals of a given symmetry, which can be populated to give high-spin or low-spin states. The existence of designer magnetic superatoms, such as alkali atom coated V and Sc atoms with several unpaired electrons, have been addressed previously from a theoretical point of view [14-16]. The focus of the present work is on the superatom nature of 13-atom Pt clusters, with chemisorbed hydrogen, based on previously-reported experimental results. This reveals a number of new aspects of magnetism which have so far not been dealt with in detail.

\section{The Concept of Superatoms}

Chemists have devised various concepts to describe properties of different classes of matter, such as atoms, molecules, transition metal complexes, salts or metals. These concepts represent specific languages with more or less fixed terminologies which facilitate communication throughout the community of chemists. The terminologies permit properties of each of these classes of matter to be explained with minimum effort. For example, for molecules, we use the concept of electron pairs which are represented by short dashes located between or on atoms depending on whether they describe 
bonding or localized non-bonding electron pairs. Flipping these dashes is a convenient way to describe chemical reactions, and every chemist understands immediately what is meant. Metals and semiconductors are described by the concept of band structures which represent a high density of delocalized molecular orbital states which extend over the entire specimen and accommodate the valence electrons; alternatively they are partly filled or nearly empty and host the conduction electrons. For transition metal complexes we have the concept of crystal field theory, which is suitable for the description of spectroscopic or magnetic properties and bonding geometry.

Clusters are small specimens with a countable and defined number of member atoms or molecules. The question is which of the concepts is suitable to describe the properties of a cluster consisting of atoms of an element which in the bulk is a metal. The density of states in a band scales approximately with the number of member atoms, so it is more than 22 orders of magnitude lower for a 13-atom cluster than for a macroscopic lump of metal consisting of one mol of atoms. How many atoms are needed to make a metal? In recent times, the phenomenon of a size-induced non-metal-to-metal transition has received considerable attention [17]. The answer depends on the element and on the temperature. Sodium, for example, has a quite continuous spectrum of optical transitions already with eight atoms [18], while for mercury one needs on the order of 400 atoms to close the s-p band gap [19].

There are basically two ways to describe these clusters. A first approach that is based on the jellium model does not explicitly take account of the atomic cores and replaces them, phenomenologically, with a square well or a parabolic potential which confines the valence electrons to the cluster volume [20]. While this picture is obviously only approximate it is relatively simple to be solved mathematically, and the solutions for the energy eigenvalues and wavefunctions are straightforward to interpret. An alternative description treats the cluster as a molecule, which makes it accessible to standard quantum chemical procedures. The atomic valence orbitals combine to give molecular orbitals which extend over the entire cluster. A consequence of the non-Coulomb potential is that the orbital angular momentum $\ell$ is no longer restricted to values $\ell<n$. This allows for orbitals of e.g., $1 \mathrm{p}, 1 \mathrm{~d}, 1 \mathrm{f}$ character, in addition to $1 \mathrm{~s}$ of the hydrogen-like wavefunctions. It has the effect that, compared with conventional atoms, a smaller fraction of cluster valence electrons has s-character.

The term superatom was coined by Watanabe and Inoshita [21] to describe the electron distribution in core-shell semiconductor nanostructures. The concept was further pioneered in chemistry by Castleman to describe entities which exhibit pronounced stability [22]. A striking example is the $\mathrm{Al}_{13}{ }^{-}$ cluster anion which has 40 valence electrons and forms a closed p-shell of strictly icosahedral symmetry, while the neutral $\mathrm{Al}_{13}$ cluster is an open-shell system with a structure which is slightly Jahn-Teller distorted $[23,24]$. The $\mathrm{Al}_{13}{ }^{-}$ion forms a halogen-like molecular ion with an iodine atom. It has an $\mathrm{Al}_{13}-\mathrm{I}$ bond energy of $2.46 \mathrm{eV}$, and the remarkable finding is that, as a consequence of a quantum size effect, the negative charge remains on the aluminum cluster. A second example is the composite superion $\mathrm{AlPb}_{12}{ }^{+}$, where 12 lead atoms are placed in a perfectly icosahedral shell around a core $\mathrm{Al}$ atom. The 50 valence electrons occupy molecular orbitals which are delocalized over the cluster and remind very much of conventional hydrogen-like atomic orbitals [25].

The concept assumes that a superatom mimics an element in the periodic table in that its properties are best understood by analogy to those of a conventional atom. Superatoms have the ability to act as relatively stable building blocks of larger assemblies that can form bonds to normal atoms or to other superatoms [22]. Furthermore, if each atom contributes one valence electron to the superatom orbitals, 
a periodic table of superatoms can be drawn in which the atomic number is directly the number of member atoms. This has been exemplified for $\mathrm{Na}_{N}$ clusters [1], and it has been shown that electron affinities of $\mathrm{Au}_{N}$ clusters show similar progressions as the ones which are well-known from the conventional periodic table of elements [26]. Without focusing on magnetism, the concept of superatoms has been applied to the understanding of ligand-protected and ligand-free gold clusters [27,28], and for thiol-ligated $\mathrm{Au}_{25}$ clusters reversible redox-induced switching between a non-magnetic and a paramagnetic modification was revealed by means of EPR spectroscopy [29].

Due to its near-spherical symmetry, the superatom character is also analogous to the well-known incompletely-filled d-electron systems, as illustrated in Figure 1 for the example of a $\mathrm{Pt}^{3+}$ ion, which is $\mathrm{a} \mathrm{d}^{7}$ system. The free ion has five degenerate $\mathrm{d}$ orbitals which host the seven electrons with maximum multiplicity, according to Hund's rules. For three unpaired electrons this results in a quartet high-spin state (Figure 1a). The situation is basically the same in an assumed weak octahedral crystal or ligand field where the environment-induced energy splitting $\Delta$ is small compared with the spin pairing energy (Figure $1 \mathrm{~b}$ ). When $\Delta$ increases and exceeds the spin pairing energy (typically $1 \mathrm{eV}$ in transition metal ions, [30]) this leads to a low-spin doublet state, as displayed in Figure 1c.

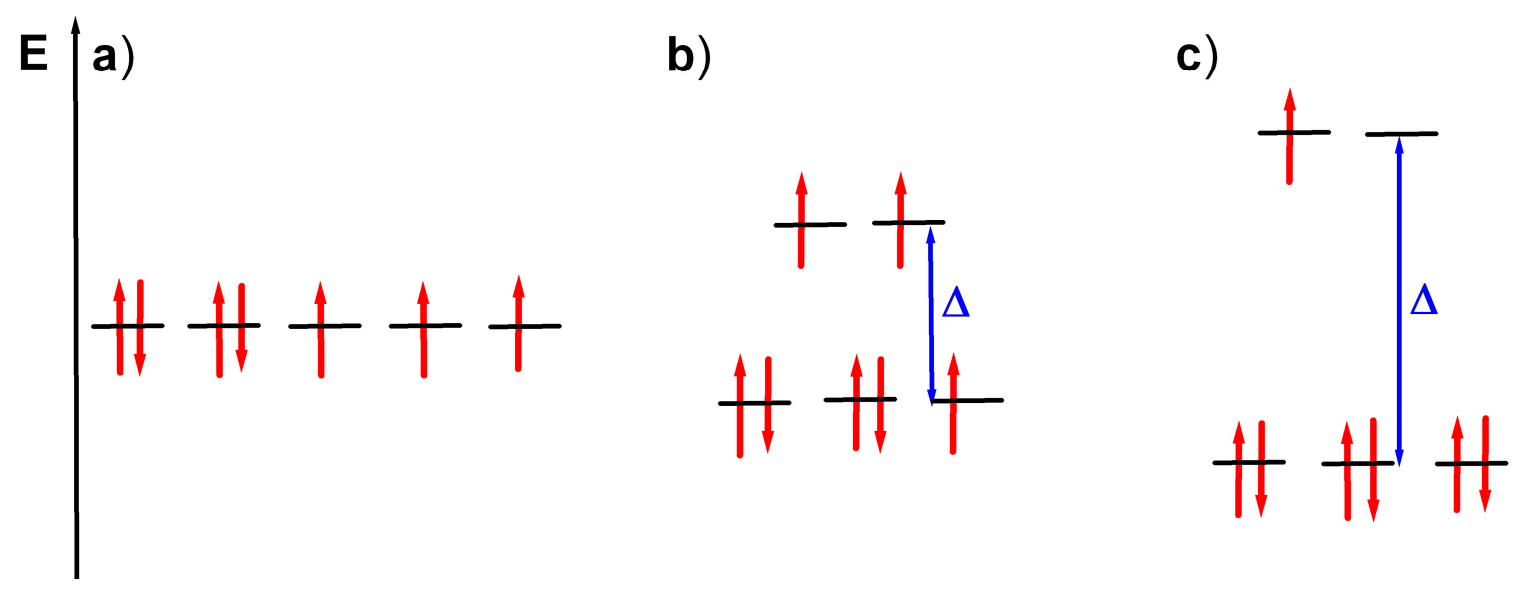

Figure 1. d-Orbital electron configuration of a $\mathrm{d}^{7}$ system (e.g., $\mathrm{Pt}^{3+}$ ) as a free ion (a) and in an assumed octahedral ligand field with small (b) and large splitting value $\Delta$ (c) leading to high-spin and low-spin character.

In heavy atoms, like Pt, the spin-orbit coupling is much more important than the crystal field splitting. For the $5 \mathrm{~d}^{9} 6 \mathrm{~s}^{1}$ triplet ground state the spin-orbit splitting between the $\mathrm{d}_{5 / 2}$ and the $\mathrm{d}_{3 / 2}$ states amounts to $5 / 2 \xi$, where $\xi$ is the spin-orbit coupling constant that was reported to amount to $1.0 \mathrm{eV} \mathrm{[31].}$

For the superatom the situation is quite analogous to that of the transition metal ion. In the simple jellium model, the $5 \mathrm{~d}^{9} 6 \mathrm{~s}^{1}$ valence electrons of atomic Pt occupy superatom states with $\mathrm{s}, \mathrm{p}, \mathrm{d}, \mathrm{f}, \mathrm{g}$, and $\mathrm{h}$ character, corresponding to cluster molecular orbitals with angular momenta $\ell=0,1,2,3,4$, and 5 [32,33]. More adequate DFT calculations for an icosahedral $\mathrm{Pt}_{13}$ cluster yield the energy levels shown in Figure 2 (left) [34]. This high symmetry will pertain only to closed-shell systems with no net spin or orbital moment and in the absence of distortions due to external influence. Open-shell systems undergo spin-orbit coupling with $\xi \approx 0.60 \mathrm{eV}$ in the d-shell, somewhat less than in the atom [34]. It affects the energies of Pt cluster orbitals by up to $2.5 \mathrm{eV}$ while the effect of Jahn-Teller distortions is smaller by two orders of magnitude [34]. Furthermore, the impact by the environment in the zeolite 
pores, such as the charge balancing protons and alkali ions, influences the energy levels and may break any residual symmetry and disturb significantly the clear shell structure of the symmetric species [32]. This leads to a varying energetic structure with a density of 6-8 states per $1 \mathrm{eV}$ around the Fermi energy of $\mathrm{Pt}_{13}$ clusters $[33,34]$. The maximum possible multiplicity is, thus, limited only by the number of states near the Fermi level which are available within an energy increment that corresponds to the spin pairing interaction which was reported to be on the order of $0.3 \mathrm{eV}$ or less [9]. It is plausible that the spin pairing energy is less compared with that of the transition metal ions due to the larger spatial extension of the cluster orbitals. It should also be reminded in this context that the spin pairing energy is a Coulomb repulsion rather than a magnetic interaction term-electrons with parallel spins populate orthogonal orbitals which allows them to avoid each other better than paired spins can in the same orbital.

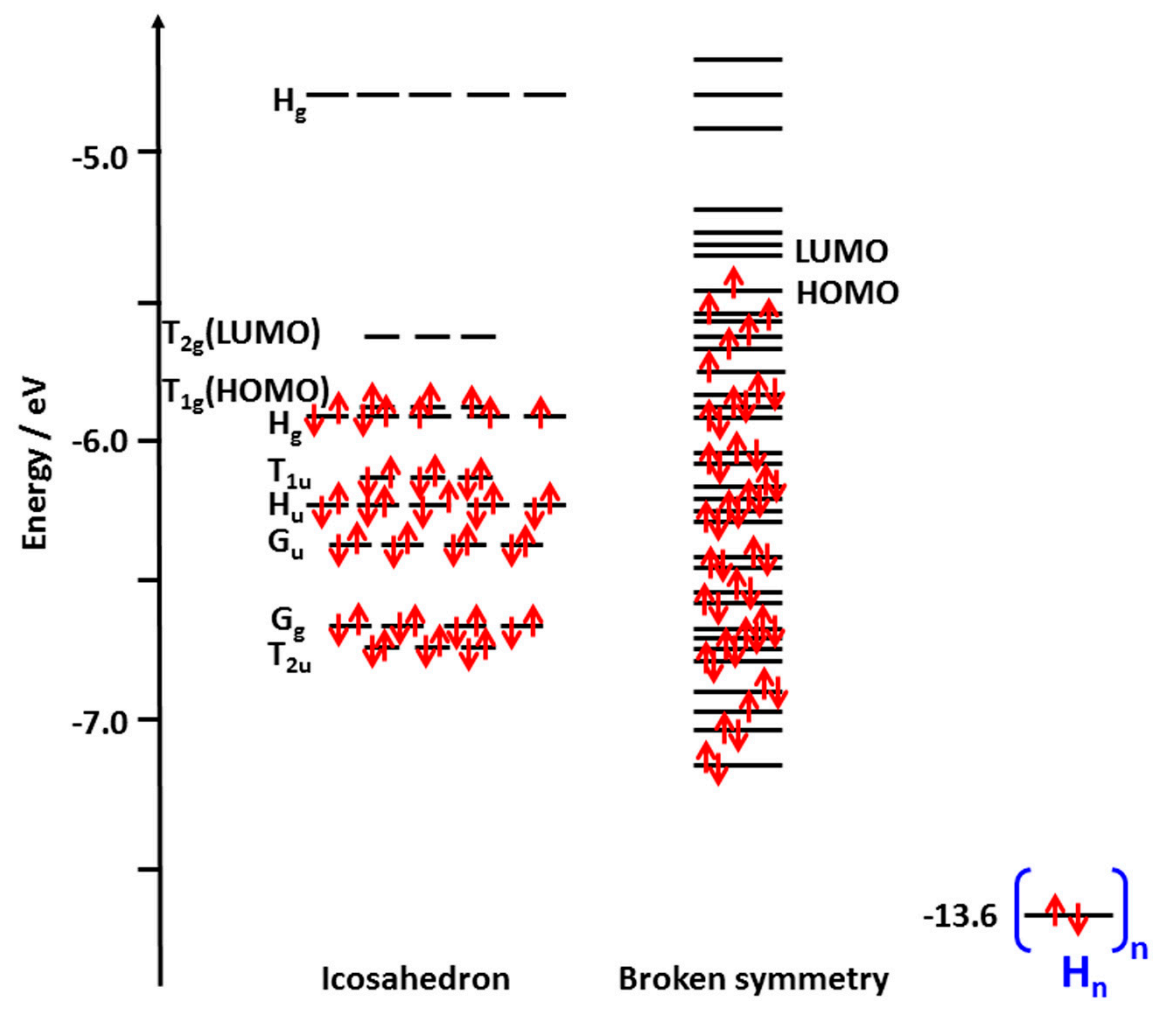

Figure 2. Calculated valence orbital energy levels of $\mathrm{Pt}_{13}$ superatom clusters in icosahedral structure (left) [34], and schematic presentation in the presence of broken symmetry (center). The ionization potential of the free $\mathrm{H}$ atom is $-13.6 \mathrm{eV}$; cluster chemisorbed $\mathrm{H}$ (right) will therefore have significant hydride character.

Unlike the surfaces of bulk Pt crystallites which can bind a single $\mathrm{H}$ atom per surface $\mathrm{Pt}$, the $\mathrm{Pt}_{13}$ clusters can bind up to $38 \pm 2$ hydrogen atoms [35], i.e., $3 \mathrm{H}$ atoms per Pt, mostly by over-edge bonding of $\mathrm{H}$ to two Pt atoms, as confirmed by FTIR spectroscopy [36]. The bond ionicity depends critically on the ionization potential of the two bonding partners, $13.6 \mathrm{eV}$ for $\mathrm{H}$ and $9.0 \mathrm{eV}$ for atomic $\mathrm{Pt}$, leading to clear hydridic bonds. By comparison, the ionization potential of the $\mathrm{Pt}_{13}$ cluster is only 5.5-6.0 eV (Figure 2, [34]), which leads to even more pronounced hydridic character of the Pt-H fragments. The Fermi level of the system is nearly independent of the amount of chemisorbed hydrogen [33]. Electronic structure calculations reveal a clear shell structure that confirms the superatom character of $\mathrm{Pt}_{13}$, as well as of the H-covered clusters [33]. 
Metal clusters are intermediate between individual atoms and bulk metals, which is seen best by the ionization potentials and the electron affinities. For the bulk metal, the two values coincide at $5.3 \mathrm{eV}$ and are called work function, while the atomic electron affinity is tabulated at $2.1 \mathrm{eV}$. Ionization potential and electron affinity represent the ability to donate or accept electrons, which is of utmost importance not only in redox reactions but also in bond formation. The above numbers demonstrate that these essential parameters vary with the size of the specimen by an amount that is much larger than the thermal energy at chemically relevant temperatures $(\approx 25 \mathrm{meV}$ at $300 \mathrm{~K})$. From a chemical point of view this is the most important origin of nanosize effects [26].

\section{Results and Discussion}

\subsection{The EPR Spectrum of Pt $t_{13} H_{m}$ Clusters}

The $\mathrm{Pt}_{13}$ clusters have been prepared and investigated in detail as a function of hydrogen or deuterium coverage in the pores of $\mathrm{NaY}$ and of KL zeolite. A fraction $<1 \%$ of the clusters is EPR active and displays a highly symmetric multiplet, which is shown in Figure 3 for the deuterated case because of the higher resolution. The spectrum can be simulated based on 12 equivalent Pt nuclei which lead to the regular multiplet structure with an isotropic Pt hyperfine coupling constant of $68.1 \mathrm{G}(227 \mathrm{MHz}$ at the given $g$ value) [37]. These simulations take into account the statistical distribution of the Pt nuclear spins in the cluster, i.e., ${ }^{195} \mathrm{Pt}(I=1 / 2$, natural abundance 33.8\%) and all other Pt isotopes with $I=0$. Since we have clusters with even, and others with odd, numbers of nuclear spin- $1 / 2$ the spectrum represent a superposition of sub-spectra with a line in the center and others with a gap in the center. The deuterium coupling is much smaller, contained in the line width and therefore not resolved by this method.

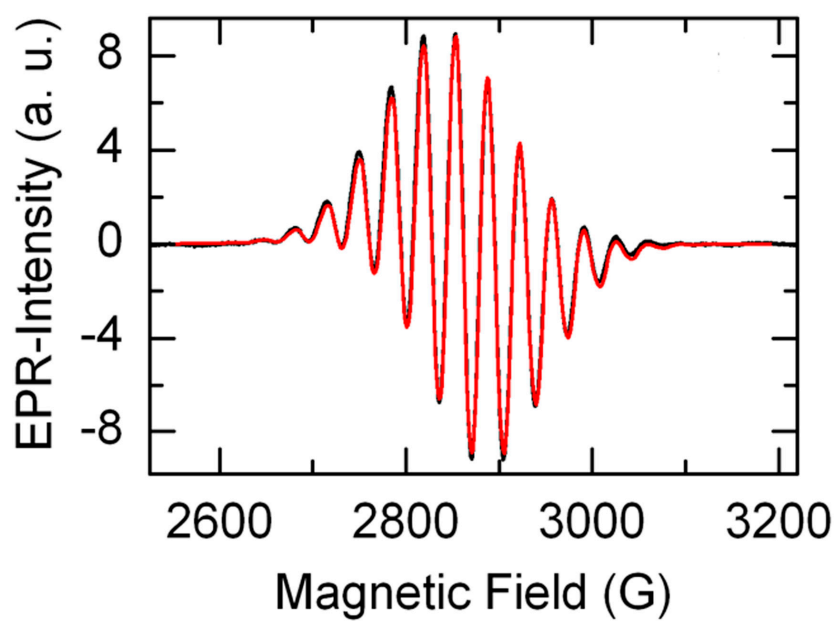

Figure 3. Experimental (black line) and simulated (red) EPR spectra of $\mathrm{Pt}_{13} \mathrm{D}_{x}$ clusters at unspecified partial deuterium coverage.

Experimental spectrum and simulation are barely distinguishable in Figure 3. The equivalence of $12 \mathrm{Pt}$ atoms is direct evidence for the high symmetry of the clusters. So where is atom 13? It is in the cluster center where only s-type superatom orbitals have significant spin density, but all higher orbitals have a node in the center, as in simple atoms. Furthermore, we remind of the fact mentioned above that superatoms have a higher fraction of non-s-type orbitals since the orbital quantum number $\ell$ is not 
restricted to values $<n$. The absence of a splitting by the 13th atom is therefore direct evidence of this superatom character of the cluster orbitals.

While the Pt hyperfine parameters are isotropic within error, the $g$ tensor of the unpaired electron shows a slight axial distortion $\left(g_{\|}=2.3795, g_{\perp}=2.3615\right.$ [37]). The significant deviation of $g$ from the free electron value $\left(g_{\mathrm{e}}=2.0023\right)$ is a consequence of spin-orbit coupling, and the $g$ anisotropy reflects the symmetry of the orbital containing the unpaired electron. All but the s-type orbitals are anisotropic, with spin density concentrated either more along a polar axis (a prolate symmetry, as e.g., in $\mathrm{p}_{z}$ ) or alternatively near the equatorial plane (oblate, as in $\mathrm{p}_{ \pm}$). Owing to the energetic proximity of different orbitals and the Jahn-Teller dynamics it is likely that the observed $g$ value represents a Boltzmann-averaged value. It is also clear that a change of hydrogen chemisorption coverage changes the population of the pseudoatom orbitals and, thus, the observed $g$ value. Instructive representations of pseudoatom molecular orbitals based on density functional calculations of closed-shell $\mathrm{AlPb}_{12}{ }^{+}$clusters which remind of familiar atomic orbitals were reported by Neukermans et al. [25].

The hydrogen hyperfine coupling can be resolved using hyperfine sublevel correlation experiments (HYSCORE), an electron-nuclear double resonance technique [37]. After initial hydrogen reduction of the $\mathrm{Pt}_{13}$ clusters the spectra reveal a single type of hydrogen with approximately axial hyperfine parameters $A_{\text {iso }}{ }^{\mathrm{H}}=12.4 \mathrm{MHz}\left(A_{\perp}{ }^{\mathrm{H}}=10.3 \mathrm{MHz}, A_{\|}^{\mathrm{H}}=16.6 \mathrm{MHz}\right)$. After complete hydrogen desorption and full readsorption to $\mathrm{Pt}_{13} \mathrm{H}_{38}$ two different types of hydrogen atoms are observed, both of axial hyperfine symmetry, with $A_{\text {iso }}{ }^{\mathrm{H} 1}=13.3 \mathrm{MHz}\left(A_{\perp}{ }^{\mathrm{H} 1}=11.7 \mathrm{MHz}, A_{\|}{ }^{\mathrm{H} 1}=16.6 \mathrm{MHz}\right)$ and $A_{\text {iso }}{ }^{\mathrm{H} 2}=6.6 \mathrm{MHz}\left(A_{\perp}{ }^{\mathrm{H} 2}=4.7 \mathrm{MHz}, A_{\|}{ }^{\mathrm{H} 2}=10.3 \mathrm{MHz}\right)$. The different types of $\mathrm{H}$ reflect the fact that a coverage of $>30 \mathrm{H}$ per cluster cannot be realized with a single type of binding site of $\mathrm{H}$, but it may be complicated by a structural transition between near icosahedral and cuboctahedral symmetry [37]. It also reveals the presence of a structural history dependence which is not fully understood, but encountered clearly again in the magnetization measurements reported below.

The above numbers for the isotropic hyperfine coupling of hydrogen can be used for a rough estimate of the spin density distribution. The value for a free $\mathrm{H}$ atom is $1420 \mathrm{MHz}$. Based on an average value of $\sim 10 \mathrm{MHz}$ per $\mathrm{H}$ the total hydrogen shell can account for on the order of $380 \mathrm{MHz}$, or less than $30 \%$ of one unpaired electron. By comparison with the tabulated isotropic hyperfine coupling of $34,410 \mathrm{MHz}$ for a free $\mathrm{Pt}$ atom [38] the isotropic value found for the $\mathrm{Pt}_{13}$ cluster corresponds to a total $\mathrm{Pt} \mathrm{s}$ orbital unpaired electron population of only $7.9 \%$, suggesting that more than $60 \%$ of the unpaired electrons must occupy Pt $\mathrm{p}$ and d orbitals.

\subsection{High-Spin Cluster States}

The magnetic parameters of samples can be obtained from measurements of the magnetization $M$ using a Superconducting Quantum Interference Device (SQUID) or X-ray Magnetic Circular Dichroism (XMCD). Measurements as a function of the applied magnetic field $H$ at low temperature yields a Langevin-type curve:

$$
M(H)=f N_{A} \frac{\mu}{13}\left[\operatorname{coth}\left(\frac{\mu H}{k_{B} T}\right)-\frac{k_{B} T}{\mu H}\right]+\chi_{0} H
$$


where $\mu$ is the magnetic moment of the cluster which represents a fraction $f$ of all Pt atoms in the sample, $k_{B}$ is the Boltzmann constant, $T$ the temperature, and $\chi_{0}$ a temperature-independent susceptibility. The curves show no hysteresis, revealing a super-paramagnetic state. Two representative measurements are shown in Figure 4 and compared with analogous measurements for Pt nanoparticles of different size. The average spin state of the particles is obtained from the curvature, and the total magnetization of the sample which, at known loading, gives the average magnetic moment per atom [11]. At low magnetic field, in the linear range, the spin state of the particle is also obtained from the temperature dependence. The saturation magnetization at high fields provides the fraction $f$ of $\mathrm{Pt}$ that contributes to magnetization. It is seen that the saturation magnetization decreases with increasing size but, on top of this, the fraction $f$ of magnetic $\mathrm{Pt}_{13}$ and $\mathrm{Pt}_{13} \mathrm{H}_{\mathrm{m}}$ clusters is $<1$.

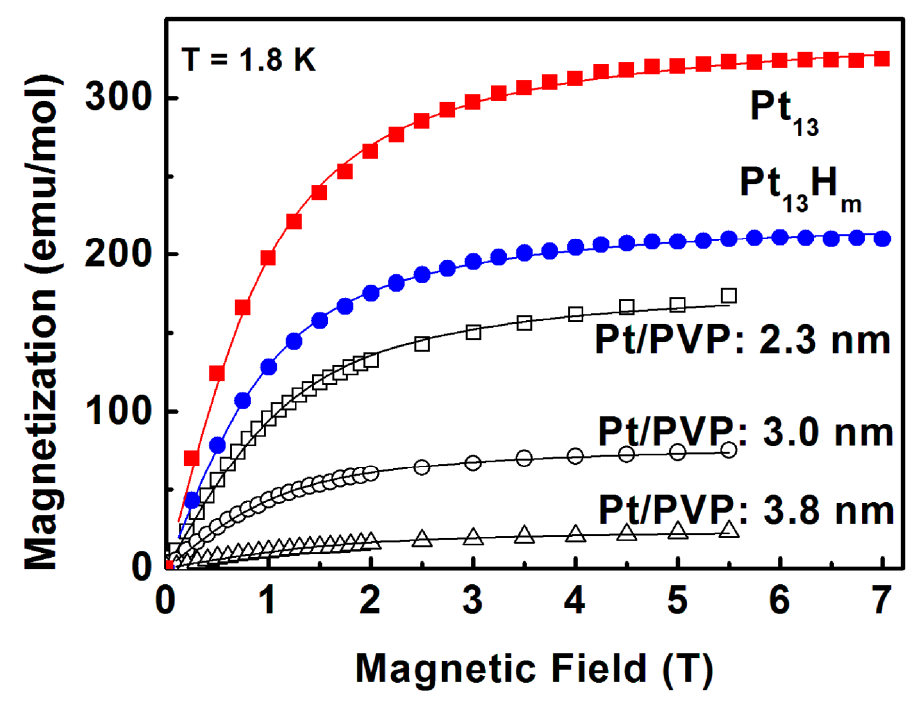

Figure 4. Langevin-type magnetization functions from SQUID measurements of $\mathrm{Pt}_{13} \mathrm{H}_{\mathrm{m}}$ clusters [10] and Pt nanoparticles in PVP polymer [2]. The magnetic moment per cluster is obtained from the curvature, the total magnetization from the plateau saturation value. The magnetization per mol of $\mathrm{Pt}$ atoms increases with decreasing particle size and depends on surface coverage with hydrogen. Because of the small fraction $f$ of high-spin $\mathrm{Pt}_{13} \mathrm{H}_{\mathrm{m}}$ clusters the plateau value for the blue and red curves appear low by a factor 5-7.

The SQUID measurements provide integral-type information, so the magnetization of the zeolite support has to be subtracted. This is particularly important when magnetic impurities are present, such as traces of iron in zeolites. XMCD is performed near the absorption edge of a chosen element (here Pt); it is, therefore, selective and does not suffer the disadvantage of SQUID measurements.

The results of both kinds of measurements are displayed in Table 1 and compared with the results for bulk Pt. The samples for the SQUID and XMCD methods were of the same type but not identical, which may introduce some additional uncertainty in the results. It is nevertheless clear from the high values of $\mu$ that the clusters are high-spin states, and if the entire magnetic moment is due to unpaired electrons with $1 \mu$ B each this is equivalent to $4-6$ electrons, or a spin state $S=2-3$ [11]. By reference to the superatom character illustrated in Figure 2 it is straightforward to understand how such large numbers of unpaired electrons can exist in these systems. As with the EPR active cluster where the spin density on the central atom was found to be below the experimental detection limit it is thought that also in the 
high-spin clusters the spin resides preferentially on the surface atoms. This is a general trend in low-dimensional systems [1], and it is in line also with the fact that the magnetic moment per atom decreases with increasing size of Pt nanoparticles [2].

Table 1. Magnetization parameters $\mu$ for high-spin platinum clusters in $\mathrm{NaY}^{\mathrm{a}}$, their fractions $f$, and the average atomic spin and orbital magnetic moments $m_{S}$ and $m_{L}$.

\begin{tabular}{cccccc}
\hline \multirow{2}{*}{ Property } & \multicolumn{2}{c}{ SQUID Measurements, 1.8 K } & \multicolumn{3}{c}{ XMCD Measurements, 7 K } \\
\cline { 2 - 6 } & $\mathbf{P t}_{\mathbf{1 3}}$ & $\mathbf{P t}_{\mathbf{1 3}} \mathbf{H}_{\mathbf{m}}$ & $\mathbf{P t}_{\mathbf{1 3}}$ & $\mathbf{P t}_{\mathbf{1 3}} \mathbf{H}_{\mathbf{m}}$ & $\mathbf{P t ~ F o i l ~}$ \\
\hline \multirow{2}{*}{$\mu / \mu_{\mathrm{B}}$ per $\mathrm{Pt}_{13}$} & $5.9(1)$ & $5.6(1)$ & & & \\
& $5.8(6)^{\mathrm{b}}$ & $8.4(6)^{\mathrm{b}}$ & $3.7(4)$ & $3.0(4)$ & - \\
\hline$f / \%$ & $14(1)$ & $10(1)$ & $14(10)$ & $20(10)$ & - \\
\hline $10^{3} m_{L} / \mu_{\mathrm{B}}$ & - & - & $5.49(9)$ & $6.39(7)$ & 2.1 \\
\hline $10^{3} m_{S} / \mu_{\mathrm{B}}$ & - & - & $17.1(6)$ & $21.8(6)$ & 0.38 \\
\hline$m_{L} / m_{S}$ & - & - & $0.32(2)$ & $0.29(2)$ & 0.38 \\
\hline
\end{tabular}

a From magnetic field dependent measurements, $T=7 \mathrm{~K}$, reference [11]; ${ }^{\mathrm{b}}$ From temperature dependent measurements at $1.8-120 \mathrm{~K}, H=0.2 T$, reference [10].

The unique feature of XMCD is that it can distinguish between the spin and atomic orbital magnetic moment, in particular the ratio $m_{L} / m_{S}$ is well defined, better than the absolute values of the individual moments which represent an average over all Pt atoms in the sample. It is significant that the ratio is lower for the clusters than in bulk Pt, but it is the largest value that has ever been observed for any $\mathrm{Pt}$ compound [39]. Relativistic calculations have confirmed these high values for the magnetic clusters and predicted them to be, by and large, independent of structural details [39].

Based on Figure 2 one expects that the cluster magnetic moment will oscillate as a function of hydrogen coverage. This is indeed the case and was reported, but the amplitudes of the oscillations were not as pronounced as expected, and the magnetization was, to some extent, history-dependent and changed over months [37]. Obviously, some slow structural dynamics, perhaps involving different environments (protons, or intrinsic exchangeable alkali cations, which balance the negative charge of the $\mathrm{Al}$ containing framework) operates. Furthermore, it is likely that there is always a distribution of clusters with different hydrogen coverages so that the oscillations are smeared out.

\subsection{EPR Observation of a Blocking Temperature}

Figure 5 shows a high-resolution transmission electron microscope image (HR-TEM, left) and the temperature dependence of the integrated intensity of the EPR multiplet of a $\mathrm{Pt}_{13} \mathrm{D}_{x}$ sample in Pt/KL zeolite with no further specified intermediate deuterium coverage (right). Interestingly, the magnetization curves following cooling in zero field (ZFC) and in the EPR field (FC at ca. $2900 \mathrm{G}$ ) split below $T_{\mathrm{b}}=14 \mathrm{~K}$. The observation of a splitting or blocking temperature is typical for single-domain superparamagnetic nanoparticles which undergo a transition to a blocked state where the Néel relaxation time of magnetization $\tau_{\mathrm{N}}$ becomes slow compared to the time scale $\tau_{\mathrm{m}}$ of a particular method of measurement. Below $T_{\mathrm{b}}$ the magnetization is frozen and depends on the magnetic field during cooling. The Néel relaxation is activated because of the nanoparticles magnetic anisotropy and given by the relation [40]: 


$$
\tau_{N}=\tau_{0} \exp \left(\frac{K V}{k_{B} T}\right)
$$

where $\tau_{0}$ is the Arrhenius pre-exponential factor, typically $10^{-9}-10^{-10} \mathrm{~s}$ (the X-band EPR frequency in the present experiment corresponds to $10^{-10} \mathrm{~s}$ ), $K$ is the magnetic anisotropy constant, $V$ the nanoparticle volume, and $K V$ the Arrhenius activation barrier between two easy directions of a single superspin magnetization. At the blocking temperature, $T=T_{\mathrm{b}}, \tau_{\mathrm{N}}$ is set equal to $\tau_{\mathrm{m}}$, and Equation (2) becomes:

$$
\tau_{m}=\tau_{0} \exp \left(\frac{K V}{k_{B} T_{b}}\right) \text {, or: } K V=k_{B} T_{b} \ln \left(\tau_{m} / \tau_{0}\right)
$$

For typical laboratory experiments, $\ln \left(\tau_{\mathrm{m}} / \tau_{0}\right)$ is on the order of 25 [41]. For the observed blocking temperature, $T_{\mathrm{b}}=14 \mathrm{~K}$, we obtain an activation barrier $K V$ of $0.30 \mathrm{eV}$, which coincides with the reported spin pairing interaction [9] and is on the same order as the average splitting of energy levels with 6-8 states per eV near the Fermi energy given above. For a cluster radius of $c a$. $0.5 \mathrm{~nm}$ we obtain $K \approx 0.6 \mathrm{eV} \mathrm{nm}{ }^{-3}\left(1 \times 10^{9} \mathrm{erg} \cdot \mathrm{cm}^{-3}\right)$ which is even larger than the value of $1 \times 10^{8} \mathrm{erg} \cdot \mathrm{cm}^{-3}$ reported for nanometer size granular $\mathrm{Co}_{20} \mathrm{Ag}_{80}$ and $\mathrm{Co}_{25} \mathrm{Ag}_{75}$ (indices are percent composition) and two orders of magnitude larger than for bulk fcc cobalt $\left(4 \times 10^{6} \mathrm{erg} \cdot \mathrm{cm}^{-3}\right)$ [40], but it was pointed out that, for such small particles, the anisotropy energy is more a surface than a volume property [41].
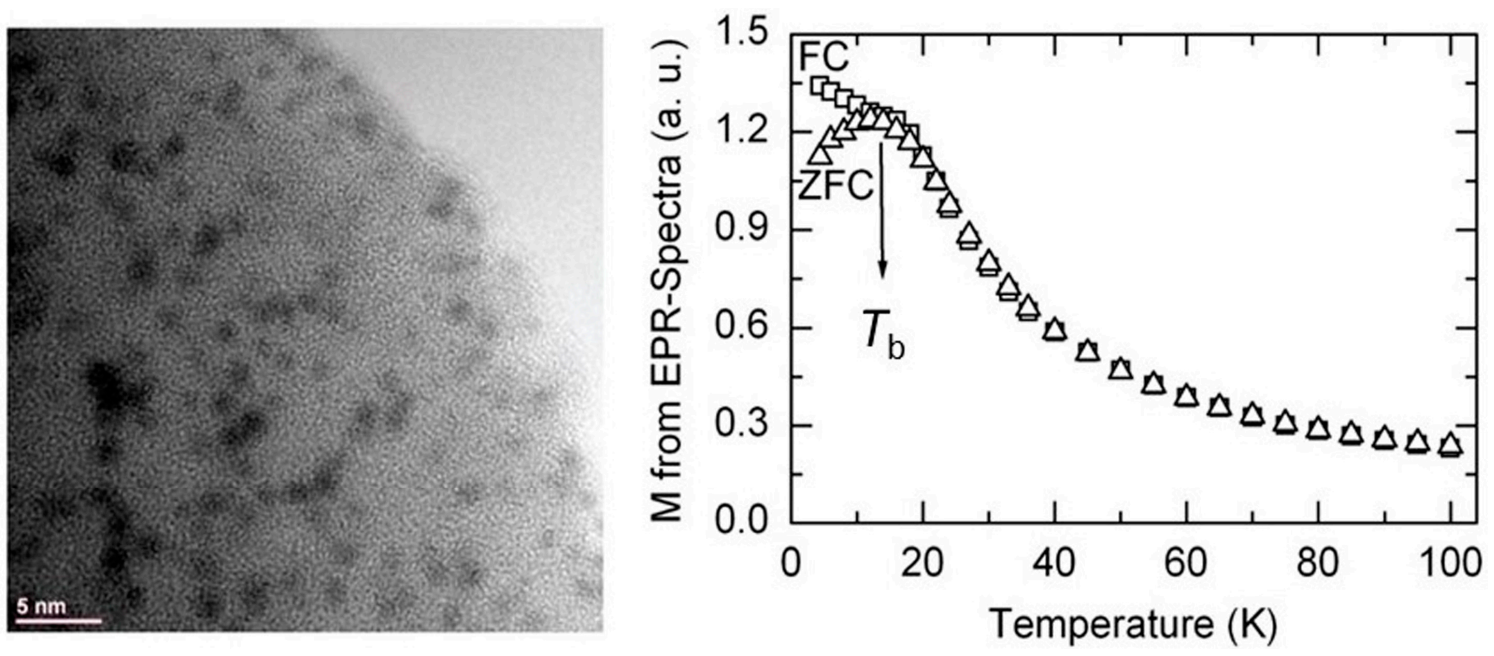

Figure 5. HRTEM image of 5.3 wt. \% Pt/KL zeolite ((left), the size bar is $5 \mathrm{~nm}$ ) and magnetization derived from the intensity of the multiplet of EPR-active $\mathrm{Pt}_{13} \mathrm{D}_{x}$ after gentle deuterium desorption (right). EPR measurements are on increasing temperature following zero-field cooling (ZFC) and field cooling (FC) to $4.5 \mathrm{~K}$.

It appears that it is straightforward to explain the temperature-dependence of the magnetization in Figure 5 based on what is known about classical superparamagnetic nanoparticles with a single macroscopic superspin. However, it has to be pointed out that the magnetizations observed here are not derived from SQUID measurements; rather they are based on EPR experiments which relate to transitions of single electron spins with an admixed orbital component. They occur at the energy of X-band microwave irradiation at $9.5 \mathrm{GHz}$ which corresponds to only $0.04 \mathrm{meV}$ at a magnetic field of $0.29 \mathrm{~T}$, less by a factor of $c a$. 3.5-7 than the average level splitting or spin pairing energy. These excitations are within the corresponding sublevels of Figure 1b,c and are not across the gap described 
by $\Delta$, and the corresponding spin states are not frozen below $T_{\mathrm{b}}$ but show biexponential relaxation behavior with time constants on the order of 1.5 and $14 \mu \mathrm{s}$ [42] and not $\approx 1 \mathrm{~s}$ at $T=T_{\mathrm{b}}$ as expected based on Equation (3).

It is not easy to find a consistent interpretation of the low temperature splitting of a ZFC and a FC branch of the EPR magnetization. To our knowledge, this splitting does not occur for individual (non-composite) paramagnetic ions. At a Pt loading of 5.3 wt. \% in KL zeolite, the concentration of monodisperse clusters is diluted, with only one $\mathrm{Pt}_{13}$ per 17 unit cells (total of $42 \mathrm{~nm}^{3}$ ), so although a certain clustering is observed in Figure 5 (left) it is not expected that there is a significant fraction at a distance at which they interact magnetically. Spin- $1 \frac{1}{2}$ states should, thus, display pure Curie behavior without any blocking temperature or magnetic ordering, so the spectra must represent high-spin states. Furthermore, the EPR spectrum of a particular sample changes as a function of temperature only in amplitude, but not in its nature, not even in line width, and the magnetic anisotropy inferred from the spectrum is very small. Spin-orbit coupling is expected to be quenched only in much higher fields. We are left to suggest that the state frozen under ZFC conditions is mainly a low-spin state, while under FC there is a higher fraction of high-spin states, and the derived value of $K V$ is equal to $\Delta$ in the superatom.

It should be noted that the SQUID measurements which were conducted for defined hydrogen coverages, all after ZFC, exhibit a maximum of magnetization similar to the one displayed in Figure 5 (right), but with $T_{\mathrm{b}}$ varying slightly in the range $6-14 \mathrm{~K}$. Only at the highest coverage of $\mathrm{Pt}_{13} \mathrm{H}_{38}$ of the otherwise identical sample there was no maximum but still not clean Curie behavior below $8 \mathrm{~K}$ [37], corroborating also that the decrease towards the lowest temperatures is not due to antiferromagnetic interaction between the different clusters. Probably, this highest coverage had a large fraction of spin- $1 / 2$ clusters.

\subsection{Diamagnetic Clusters}

Approximately $15 \%$ of the clusters were found to be of high-spin, and $<1 \%$ spin- $1 / 2$. This leaves a fraction of $c a$. $85 \%$ for diamagnetic clusters. The question is why we have different types of clusters which are essentially all composed of 13 atoms, and all roughly of the same structure. The answer is, of course, that there are differences. First of all, the nominal hydrogen coverage is likely an average value, except for the highest loading (38 H per cluster), and the completely H-desorbed state. Secondly, even nominally-identical clusters are found in different environments in the zeolite pore since a varying number of remaining alkali ions from the partial exchange with Pt salt will be found in the same pore as the cluster. Some of them may have been replaced by protons and, furthermore, it cannot be excluded that some of the water produced during PtO reduction by hydrogen has remained in the polar environment under the relatively mild treatment conditions. Thus, there is a variety of environments which act like ligands on transition metal ions and further perturb the symmetry of the systems.

\subsection{Super-Diamagnetism}

As expected, based on the Curie law, the paramagnetism of the high-spin clusters dampens out quickly with increasing temperature. Surprisingly, at $300 \mathrm{~K}$, a negative magnetization is observed that is proportional with the applied magnetic field (Figure 6) [7]. The slope of the magnetization is the diamagnetic susceptibility, $\chi_{\mathrm{D}}$. Normally, this property can be calculated from atomic increments as it is 
only slightly dependent on the chemical nature; for example, the ionic charge. It is conventionally explained by induced ring currents on the atoms (Figure 7), and all types of clusters, including the paramagnetic ones, contribute. Here, however, depending on the hydrogen coverage, $\chi_{\mathrm{D}}$ is larger by a factor 36-50 than the expected tabulated value of $c a .-12 \mathrm{~cm}^{3} \cdot \mathrm{mol}^{-1}$ [7]. This enhancement is spectacular and reveals a superatom effect that has been predicted for sodium clusters [6] but experimentally observed so far only for the $\mathrm{Pt}_{13}$ clusters [7].

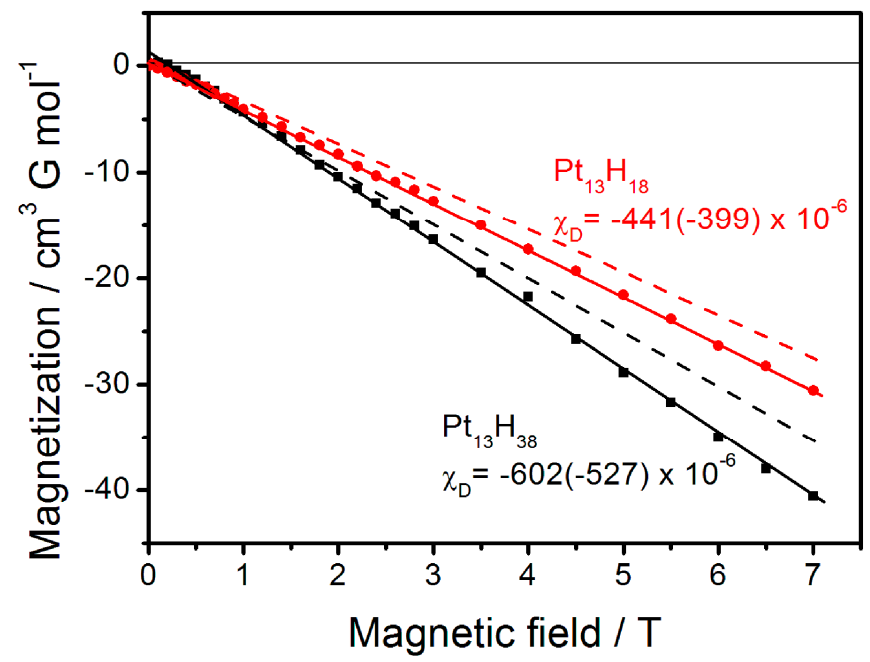

Figure 6. SQUID results of magnetization at $300 \mathrm{~K}$ of $\mathrm{Pt}_{13} \mathrm{H}_{18}$ (red) and $\mathrm{Pt}_{13} \mathrm{H}_{38}$ (black) supported in specifically synthesized iron-free KL zeolite after correction for the magnetization of the empty zeolite (symbols and full lines) compared with calculated curves (broken lines and numbers in parentheses). The numbers relate to one mol of $\mathrm{Pt}$ atoms with $\chi_{\mathrm{D}}$ in units of $\mathrm{cm}^{3} \cdot \mathrm{mol}^{-1}$.

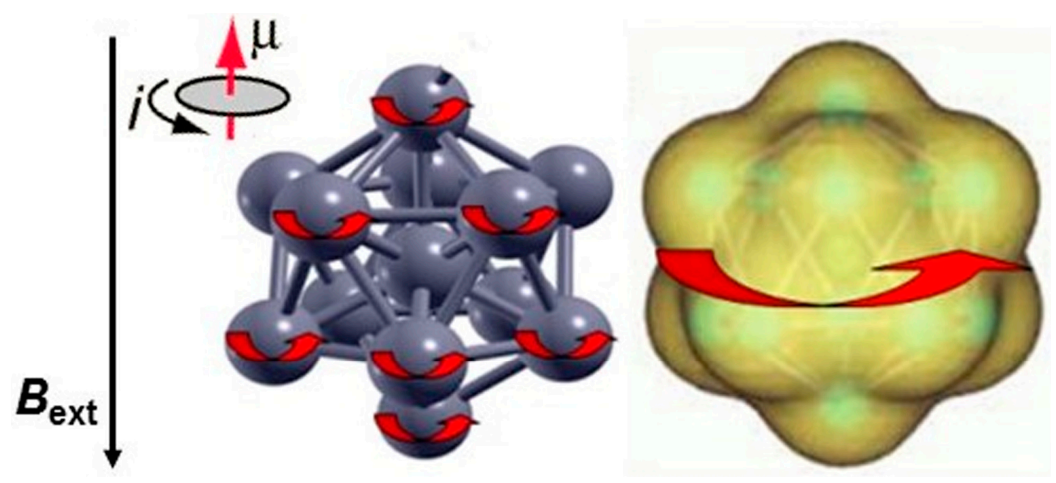

Figure 7. Induced ring currents $i$ and induced magnetic moment $\mu$ antiparallel to the external magnetic field $B_{\text {ext }}$ on individual atoms (left) and on the hydrogen covered superatom ((right), with the blue-shaded area representing the hydrogen shell). The magnitude of $\mu$ is proportional to the area of the ring and to the number of electrons involved in the ring current.

The behavior can be simulated based on the classical formula (here in S.I. units) for a cluster consisting of $N$ atoms

$$
\chi=-\frac{e^{2} \mu_{0}}{6 m_{e}} \frac{N}{V} \sum_{i}\left\langle r_{i}^{2}\right\rangle
$$


where $e$ is the electron charge, $m_{\mathrm{e}}$ the electron mass, $\mu_{0}$ the vacuum permeability, $V$ the atomic volume and $\left\langle r_{i}^{2}\right\rangle$ is the mean square distance of the $i$-th electron from the center [43]. Most remarkably, the Pt

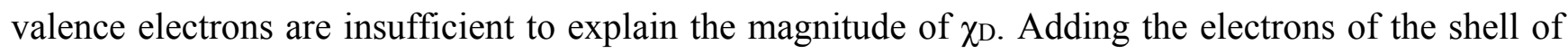
overlapping hydride ions yields a contribution of similar magnitude and improves the results considerably although they are still low by $10 \%-15 \%$ [7]. It is nevertheless remarkable that the ratio between the calculated and the experimental $\chi_{D}$ values is roughly the same for the two samples. It is expected that proper quantum mechanical evaluation of the mean square radius using the wave function of the system would improve the result further.

This is another compelling example that a cluster with electrons which are delocalized over the cluster orbitals behaves like an atom and should be regarded as a superatom.

\subsection{Potential Superconductivity}

There are earlier reports of superconductivity in compacted Pt powders of $2 \mu \mathrm{m}$ grain size with a transition temperature $T_{\mathrm{c}}$ of $1.38 \mathrm{mK}$ [44] and of $100-300 \mathrm{~nm}$ grain size with $T_{\mathrm{c}}=20 \mathrm{mK}$ [45]. The results point to higher transition temperatures and critical fields for decreasing particle size. The giant diamagnetism reported in the above Figure 6 for the $\mathrm{Pt}_{13}$ clusters was discussed previously as a possible indication of superconductivity [7], however, diamagnetism is not a sufficient argument for superconductivity. Interestingly, the granular structure of the sample does not prevent superconductivity. Theoretical work predicts even a strengthening of pair correlation in nanoclusters and a large enhancement of $T_{\mathrm{c}}$ with potential for room-temperature superconductivity [46,47]. In the present samples with a Pt loading of $6 \mathrm{wt}$. \% the clusters are too far apart for the necessary coupling, but there should be no difficulty synthesizing samples with significantly higher loadings.

Renewed interest in platinum hydride systems comes from the recent discovery of superconductivity with a transition temperature of $203 \mathrm{~K}$ in hydrogen sulfide under high pressure [48], which led to considering further hydride systems as possible superconductors. Ambiguous experimental data obtained with $\mathrm{SiH}_{4}$ were suggested to be actually due to $\mathrm{PtH}$, which sparked theoretical confirmation [49].

\section{Conclusions}

Previous discussions of superatoms focused on their electronic properties. Here we show that magnetic properties of a small system provide integral or even localized information on the system's wave function and, therefore, on their physical and chemical behavior. The magnetic properties of near-spherical 13-atom platinum clusters are shown to be well explained based on the analogy of such delocalized electronic systems with the electronic structure of isolated atoms. This superatom concept describes well the undetectably-small hyperfine coupling of the central Pt atom, the high-spin states with spin multiplicities that depend on the hydrogen coverage and, in particular, the unique phenomenon of super-diamagnetism. Depending somewhat on the extent of chemisorbed hydrogen the magnetization shows a blocking temperature in the range of 6-14 K. At higher Pt loading, the samples may be promising for observation of granular superconductivity. 


\section{Acknowledgments}

We thank J. van Slageren for useful discussions.

\section{Author Contributions}

C.J. performed and analyzed the experiments and E.R. extended the interpretation and wrote the paper.

\section{Conflicts of Interest}

The authors declare no conflict of interest.

\section{References}

1. Roduner, E. Nanoscopic Materials: Size-Dependent Phenomena and Growth Principles, 2nd ed.; Royal Society of Chemistry: Cambridge, UK, 2014; Chapter 5.

2. Yamamoto, Y.; Miura, T.; Nakae, Y.; Teranishi, T.; Miyake, M.; Hori, H. Magnetic Properties of the Noble Metal Nanoparticles Protected by Polymer. Physica B 2003, 329-333, 1183-1184.

3. Nakae, Y.; Seino, T.; Teranishi, T.; Miyake, M.; Hori, H. Anomalous spin polarization in Pd and Au nano-particles. Physica B 2000, 284, 1758-1759.

4. Moseler, M.; Häkkinen, H.; Barnett, R.N.; Landman, U. Structure and Magnetism of Neutral and Anionic Palladium Clusters. Phys. Rev. Lett. 2001, 86, 2545-2548.

5. Khanna, S.; Jena. P. Physics and Chemistry of Finite Size Systems: From Clusters, to Crystals; Jena, P., Khanna, S.N., Rao, B.K., Eds.; Kluwer Academic: Dordrecht, The Netherlands, 1992; Volume I, pp. 709-720.

6. Kresin, V.V. Electronic Structure of Small Metal Clusters: Thomas-Fermi Statistical Theory. Phys. Rev. B 1988, 38, 3741-3746.

7. Roduner, E.; Jensen, C.; van Slageren, J.; Rakoczy, R.A.; Larlus. O.; Hunger, M. Anomalous Diamagnetic Susceptibility in 13-Atom Platinum Superatoms. Angew. Chem. Int. Ed. 2014, 53, 4318-4321.

8. Schmauke, T.; Eichel, R.-A.; Schweiger, A.; Roduner, E. Electron Paramagnetic Resonance Studies of a Platinum Cluster in Linde L and Faujasite Zeolites. Phys. Chem. Chem. Phys. 2003, 5, 3076-3084.

9. Liu, X.; Dilger, H.; Eichel, R.A.; Kunstmann, J.; Roduner, E. A Small Paramagnetic Platinum Cluster in a NaY Zeolite: Characterization and Hydrogen Adsorption and Desorption. J. Phys. Chem. B 2006, 110, 2013-2023.

10. Liu, X.; Bauer, M.; Bertagnolli, H.; Roduner, E.; van Slageren, J.; Phillipp, F. Structure and Magnetization of Small Monodisperse Platinum Clusters. Phys. Rev. Lett. 2006, doi:10.1103/PhysRevLett.97.253401; Erratum in 2009, 102, 049902E.

11. Bartolomé, J.; Bartolomé, F.; García, L.M.; Roduner, E.; Akdogan, Y.; Wilhelm, F.; Rogalev, A. Magnetization of $\mathrm{Pt}_{13}$ clusters supported in a NaY zeolite: A XANES and XMCD study. Phys. Rev. B 2009, 80, 014404:1-014404:10. 
12. Longo, R.C.; Gallego. L.J. Structures of 13-Atom Clusters of FCC Transition Metals by ab initio and Semi-Empirical Calculations. Phys. Rev. B 2006, 74, 193409:1-193409:4.

13. Akdogan, Y.; Anantharaman, S.; Liu, X.; Lahiri, G.K.; Bertagnolli, H.; Roduner, E. Reconstruction of $\mathrm{Pt}_{13}$ Clusters into $\mathrm{Pt}_{2}(\mathrm{CO})_{m}$ on $\mathrm{CO}$ Addition in NaY Zeolite. J. Phys. Chem. C 2009, 113, 2352-2359.

14. Reveles, J.U.; Clayborne, P.A.; Reber, A.C.; Khanna, S.N.; Pradhan, K.; Sen, P.; Pederson, M.R. Designer magnetic superatoms. Nat. Chem. 2009, 1, 310-315.

15. King, R.B. Magnetic superatoms. Nat. Chem. 2009, 1, 260-261.

16. Pradhan, K.; Reveles, J.U.; Sen, P.; Khanna, S.N. Enhanced magnetic moments of alkali metal coated Sc clusters: New magnetic superatoms. J. Chem. Phys. 2010, 132, 124302:1-124302:5.

17. Edwards, P.P.; Johnston, R.L.; Rao, C.N.R. On the Size-Induced Metal-Insulator Transition in Clusters and Small Particles. In Metal Clusters in Chemistry; Braunstein, P., Oro, L.A., Raithby, P.R., Eds.; Wiley: Weinheim, Germany, 1999; Volume 3, pp. 1454-1478.

18. Kreibig, U.; Volmer, M. Optical Properties of Metal Clusters; Springer Series in Materials Science; Springer: Berlin, Germany, 1995; Volume 25, pp. 275-436.

19. Busani, R.; Folkers, M.; Cheshnovsky, O. Direct Observation of Band-Gap Closure in Mercury Clusters. Phys. Rev. Lett. 1998, 81, 3836-3839.

20. De Heer, W.A. The Physics of Simple Metal Clusters: Experimental Aspects and Simple Models. Rev. Mod. Phys. 1993, 65, 611-676.

21. Watanabe, H.; Inoshita, T. Superatoms: A Novel Concepts in Materials Science. Optoelectron. Devices Technol. 1986, 1, 33-39.

22. Castleman, A.W.; Khanna, S.N. Clusters, Superatoms, and Building Blocks of New Materials. J. Phys. Chem. C 2009, 113, 2664-2675.

23. Leuchtner, R.E.; Harms, A.C.; Castleman, A.W. Thermal Metal Cluster Anion Reactions: Behavior of Aluminum Clusters with Oxygen. J. Chem. Phys. 1989, 91, 2753-2755.

24. Bergeron, D.E.; Castleman, A.W., Jr.; Morisato, T.; Khanna, S.N. Formation of $\mathrm{Al}_{13 I^{-}}$: Evidence for the Superhalogen Character of $\mathrm{Al}_{13}$. Science 2004, 304, 84-87.

25. Neukermans, S.; Janssens, E.; Chen, Z.F.; Silverans, R.E.; Schleyer, P.V.R.; Lievens, P. Extremely Stable Metal-Encapsulated $\mathrm{AlPb}_{10}{ }^{+}$and $\mathrm{AlPb}_{12}{ }^{+}$Clusters: Mass-Spectrometric Discovery and Density Functional Theory Study. Phys. Rev. Lett. 2004, 92, 163401:1-163401:4.

26. Roduner, E. Size Matters-Why Nanomaterials Are Different. Chem. Soc. Rev. 2006, 35, 583-592.

27. Walter, M.; Akola, J.; Lopez-Acevedo, O.; Jadzinsky, P.D.; Calero, G.; Ackerson, C.J.; Whetten, R.L.; Grönbeck, H.; Häkkinen, H. A unified view of ligand-protected gold clusters as superatom complexes. Proc. Natl. Acad. Sci. USA 2008, 105, 9157-9162.

28. Häkkinen, H. Atomic and electronic structure of gold clusters: Understanding flakes, cages and superatoms from simple concepts. Chem. Soc. Rev. 2008, 37, 1847-1859.

29. Zhu, M.; Aikens, C.M.; Hendrich, M.P.; Gupta, R.; Qian, H.; Schatz, G.C.; Jin, R. Reversible switching of magnetism in thiolate-protected Au25 superatoms. J. Am. Chem. Soc. 2009, 131, 2490-2492.

30. Vanquickenborne, L.G.; Haspeslagh, L. On the Meaning of the Spin-Pairing Energy in Transition-Metal Ions. Inorg. Chem. 1982, 21, 2448-2454. 
31. Christensen, N.E. Spin-orbit projected d-densities of states of Pd, Ag, Pt, Au. J. Phys. F Met. Phys. 1978, 8, L51-L55.

32. Varns, R.; Strange, P. Super-Atom Properties of 13-Atom Clusters of Group 13 Elements. Phys. Status Solidi B 2012, 249, 2179-2189.

33. Watari, N.; Ohnishi, S. Electronic Structure of H Adsorbed on Pt 13 Clusters. J. Chem. Phys. 1997, 106, 7531-7540.

34. Watari, N.; Ohnishi, S. Atomic and Electronic Structures of $\mathrm{Pd}_{13}$ and $\mathrm{Pt}_{13}$ Clusters. Phys. Rev. B Condens. Matter 1998, 58, 1665-1677.

35. Jensen, C.; Buck, D.; Dilger, H.; Bauer, M.; Phillipp, F.; Roduner, E. Maximum hydrogen chemisorption on KL zeolite supported Pt clusters. Chem. Commun. 2013, 49, 588-590.

36. Keppeler, M.; Roduner, E. Platinum-Hydrogen Vibrations and Low Energy Electronic Excitations of 13-Atom Pt Nanoclusters. Phys. Chem. Chem. Phys. 2014, 16, 26613-26616.

37. Jensen, C.; van Slageren, J.; Jakes, P.; Eichel, R.-A.; Roduner, E. Support Effects on Hydrogen Desorption, Isotope Exchange, Chemical Reactivity, and Magnetism of Platinum Nanoclusters in KL Zeolite. J. Phys. Chem. B 2013, 117, 22732-22745.

38. Morton, J.R.; Preston, K.F. Atomic Parameters for Paramagnetic Resonance Data. J. Magn. Reson. 1978, 30, 577-582.

39. Buneãu, O.; Bartolomé, J.; Bartolomé, F.; García, L.M. Large Orbital Magnetic Momentum in Pt 13 Clusters. J. Phys. Condens. Matter 2014, 26, 196006:1-196006:11.

40. Zhang, I.D.; Budnick, J.D.; Hynes, W.A.; Chien, C.L.; Xiao, J.Q. Effect of the magnetic field on the superparamagnetic relaxation in granular Co-Ag samples. Appl. Phys. Lett. 1998, 72, 2053-2055.

41. Hickey, J.B.; Howson, M.A.; Greig, D.; Wiser, N. Enhanced magnetic anisotropy energy density for superparamagnetic samples of cobalt. Phys. Rev. B 1996, 53, 32-33.

42. Jensen, C. Elektronische Struktur, Magnetismus und Chemische Reaktivität von $\mathrm{Pt}_{13}$-Nanoclustern im KL-Zeolith. Ph.D. Thesis, University of Stuttgart, Stuttgart, Germany, 2013.

43. Blundell, S. Magnetism in Condensed Matter; Oxford Masters Series in Condensed Matter Physics; Oxford University Press: Oxford, UK, 2001.

44. König, R.; Schindler, A.; Hermannsdörfer, T. Superconductivity of Compacted Platinum Powder at Very Low Temperatures. Phys. Rev. Lett. 1999, 82, 4528-4531.

45. Schindler, A.; König, R.; Hermansdörfer, T.; Braun, H.F.; Esca, G.; Günther, G.; Meissner, M.; Mertig, M.; Wahl, R.; Pompe, W. Superconductivity at $20 \mathrm{mK}$ in compacted submicrometer platinum powders. Physica B 2003, 329-333, 1427-1428.

46. Kresin, V.Z.; Ovchinnikov, Y.N. Shell structure and strengthening of superconductivity pair correlation in nanoclusters. Phys. Rev. B 2006, 74, 024514:1-024514:11.

47. Kresin, V.Z.; Ovchinnikov, Y.N. "Giant" strengthening of superconducting pairing in metallic nanoclusters: Large enhancement of $T_{\mathrm{c}}$ and potential for room-temperature superconductivity. Phys. Uspekhi 2008, 51, 427-435.

48. Drozdov, A.P.; Eremets, M.I.; Trojan, I.A.; Ksenofontov, V.; Shylin, S.I. Conventional superconductivity at 203 Kelvin at high pressures in the sulfur hydride system. Nature 2015, 525, $73-76$. 
49. Szezęśniak, D.; Zemła, T.P. On the high-pressure superconducting phase in platinum hydride. Supercond. Sci. Technol. 2015, 28, 085018:1-085018:5.

(C) 2015 by the authors; licensee MDPI, Basel, Switzerland. This article is an open access article distributed under the terms and conditions of the Creative Commons Attribution license (http://creativecommons.org/licenses/by/4.0/). 